\title{
O ESTUDO DA DEGRADAÇÃO ELETROQUÍMICA DO CORANTE VERMELHO ÁCIDO PRESENTE EM EFLUENTES TÊXTEIS.
}

\author{
E. M. SALES ${ }^{1}$, E. O. VILLAR ${ }^{1}$ \\ ${ }^{1}$ Universidade Federal de Campina Grande, Departamento de Engenharia Química. \\ E-mail para contato: emanuele.montenegro@gmail.com
}

\begin{abstract}
RESUMO - A indústria têxtil é classificada como um dos setores mais poluentes dentro das atividades industriais. Este trabalho teve como objetivo a avaliação da eficiência de oxidação direta (eletroquímica) e indireta (química) do corante vermelho ácido usando um eletrodo de óxidos-semicondutores do tipo DSA $\left(\mathrm{Ti} / \mathrm{Ru}_{0,3} \mathrm{Ti}_{0,7} \mathrm{O}_{2}\right)$ para degradação de efluente têxtil sintético. Pode-se concluir que o processo de degradação eletroquímica é regido pelo controle faradaico. Verificou-se que após 20 minutos de eletrólise, observa-se uma degradação de $92,64 \%$ do corante para uma (ddp) de $3.5 \mathrm{~V}$ chegando a quase $100 \%$ de remoção de cor para uma (ddp) de 5,0V.
\end{abstract}

\section{INTRODUÇÃO}

A indústria têxtil é classificada como um dos setores mais poluentes entre as diferentes atividades humanas. Em média, um milhão de toneladas de corantes são produzidos a cada ano em todo o mundo (JOVIC et al., 2013). O que representa um impacto ambiental devido à geração de grandes volumes de resíduos onde os mesmos apresentam altas cargas de compostos orgânicos.

Neste trabalho foi identificado se o processo de degradação eletroquímica do corante é regido pela transferência de carga (controle faradaico) ou pela convecção difusão, para um reator do tipo PFR e foi investigada a influência dos principais parâmetros operacionais, tais como: potencial de oxidação, quantidade de cloreto utilizado, fluxo de alimentação, sobre a taxa de redução da concentração.

\section{MATERIAIS E METÓDOS}

O efluente sintético foi tratado através do processo de eletrólise com um tempo de duração de 1 hora sendo que para cada condição avaliada obteve-se os resultados em duplicata. Para a análise do decréscimo da concentração do corante vermelho acido realizouse a coleta de 12 alíquotas amostrais de $2 \mathrm{mLs}$ em cada experimento com um tempo de até 60 minutos. As mesmas foram analisadas posteriormente utilizando-se um espectrofotômetro da HITACHI MODELO U-1800 UV-VIS.

Foi utilizado o corante Vermelho Remazol Ácido 88. As soluções sintéticas foram preparadas dissolvendo em água destilada contendo sulfato de sódio $\left(\mathrm{Na}_{2} \mathrm{SO}_{4}\right)$ 0,5 $\mathrm{mol} / \mathrm{L}^{-1}$ para uma oxidação direta, porque este sal está presente em muitas águas residuais têxteis e apresenta boa condutividade elétrica, para oxidação indireta, foram preparadas soluções 
sintéticas dissolvendo o corante. As concentrações de corantes usadas foram de 0,2ppm para dissolver em água destilada contendo cloreto de sódio $(\mathrm{NaCl})$.

O reator eletroquímico constituído por um tubo de acrílico com diâmetro interno de $6,4 \mathrm{~cm}$ e altura de $57 \mathrm{~cm}$. A Figura 1 representa o sistema experimental constituídos dos seguintes acessórios: Célula eletroquímica (1); (2) Tanque de alimentação de 10L; (3) rotâmetro Conalt Mod 440 (vazão máxima de 1200L/h); (4) Fonte Power supply EMG 181 de 30V e 10A; (5) Inversor de frequência que controla a rotação da bomba; (6) Bomba centrífuga SCHINEIDER Mod. MCI-EF $1 / 2$ CV para recirculação da solução, tanque de alimentação com capacidade para $10 \mathrm{~L}$.

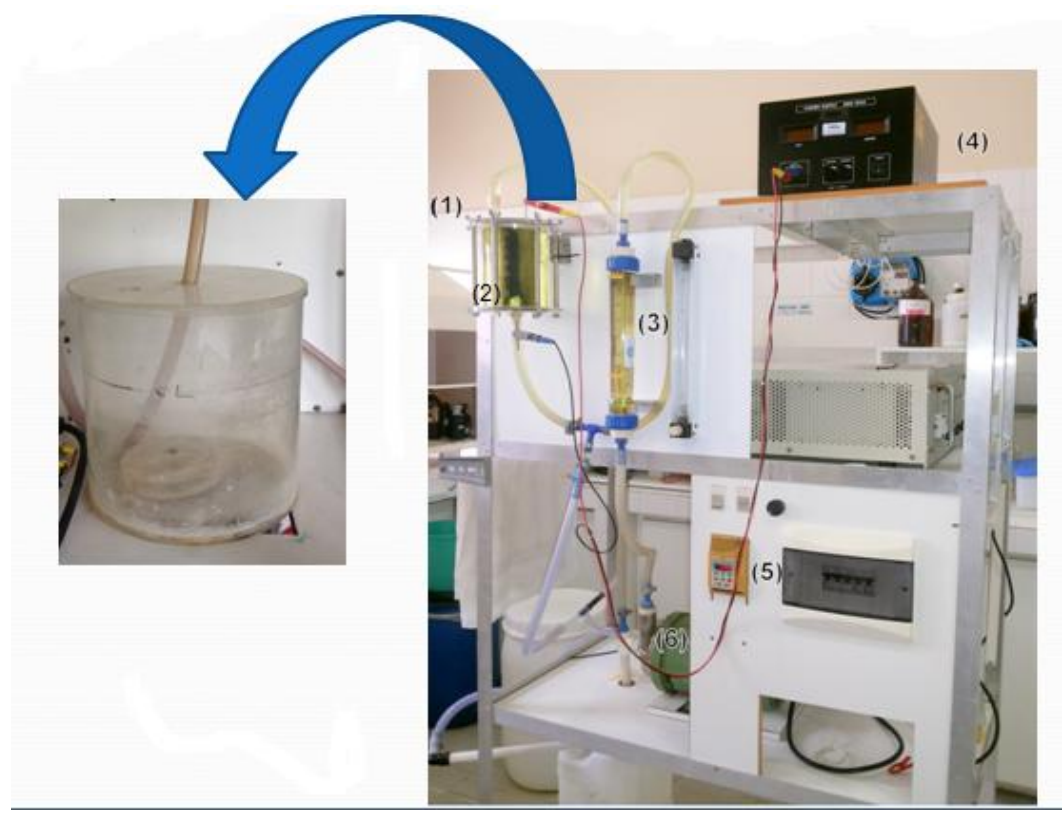

Figura 1 - Sistema do Reator Eletroquímico.

\subsection{Análise da Degradação do Corante}

Para a determinação da concentração do corante, foi primeiramente levantada uma curva de calibração, resultando na Equação (1), onde se observou um comprimento de onda máximo $\lambda=510 \mathrm{~nm}$, no qual o corante apresentava maior absorbância. Esta determinação foi realizada utilizando soluções com diferentes concentrações do corante até um máximo de 2 ppm do corante vermelho ácido. Através da determinação do comprimento de onda de máxima absorbância, fez-se uma curva padrão da absorbância versus concentração do corante. O ajuste utilizado foi o linear com coeficiente de correlação acima de 0,99.

$$
p p m=-0,0086+0,00728 \cdot A b s
$$




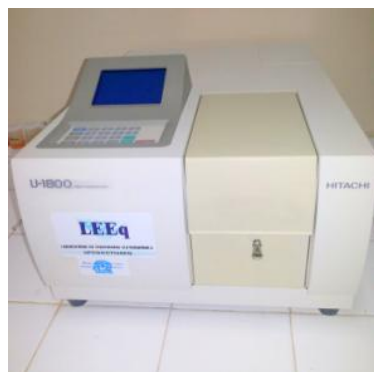

Figura 2 - Espectrofotômetro HITACHI MODELO U-1800 UV-VIS.

Experimentalmente, a redução da cor foi expressa em percentagem, conforme a Equação (2).

$$
\text { Redução da cor } \%=\left(\frac{\mathrm{C}_{\text {corante }}(\mathrm{t}=0)-\mathrm{C}_{\text {corante }}(\mathrm{t})}{\mathrm{C}_{\text {corante }}(\mathrm{t}=0)}\right) \cdot 100
$$

Para verificar se o modelo segue a cinética de degradação por regime difusional ou faradaico, foi determinado o $K_{d}^{g}$ (coeficiente de transferência de massa combinado, ou seja, com produção de gás) através da Equação (5), deduzida de um balanço de massa para um reator de fluxo pistão (PFR) com recirculação contínua. Esta expressão a taxa de remoção teórica sob-regime de controle difusional (WALSH, 1993).

$$
X_{A}^{P F R}=1-\frac{C_{t}}{C_{t 0}}=1-\exp \left\{-\frac{t}{\tau_{r}}\left[1-\exp \left(-\frac{\overline{K_{d}^{g}} \cdot A}{Q_{v}}\right)\right]\right\}
$$

Partindo da suposição que a eletro-oxidação do corante obedece a uma cinética infinitamente rápida de primeira ordem, o coeficiente de transferência de massa combinado, foi determinado a partir da coleta de amostras de uma solução eletrolítica composta por Ferricianeto e Ferrocianeto de potássio - concentrações iguais a $0,002 \mathrm{M}$ em meio alcalino $\mathrm{NaOH}(0,1 \mathrm{M})$ antes e ao final da eletrólise (60 minutos). A corrente limite $\left(\mathrm{I}_{\mathrm{d}}{ }^{\mathrm{g}}\right)$ calculada conforme Equação (4) foi determinada através da queda de concentração do ferricianeto de potássio por titulação amperométrica com cloreto de cobalto. Finalmente o coeficiente, $\overline{K_{d}^{g}}$, foi determinado conforme a Equação (5) (ElSNER (1984); LIMA (2013); ALBUQUERQUE (2006)).

$$
\begin{aligned}
& I_{d}^{g}=\frac{n \cdot F \cdot \Delta C \cdot V_{c}}{t} \\
& \overline{K_{d}^{g}}=\frac{I_{d}^{g}}{n \cdot F \cdot A \cdot C}
\end{aligned}
$$

\section{RESULTADOS E DISCUSSÕES}

Foi construída uma curva de calibração para diferentes concentrações do vermelho ácido, utilizando-se um espectrofotômetro. Cada eletrólise teve uma duração de 1 hora e todas foram feitas em triplicata. Para avaliarmos o decréscimo da concentração inicial do vermelho 
ácido 88, foram retiradas 12 alíquotas de amostras de $2 \mathrm{ml}$ para os tempos de $0,2,5,10,15$, $25,40,50,60$. As mesmas foram analisadas, posteriormente, utilizando-se um espectrofotômetro. Inicialmente a degradação do corante foi realizada a um potencial, igual a 3,5 V. Este potencial foi inicialmente escolhido por apresentar um potencial mínimo de desprendimento de cloro, evitando-se assim um consumo energético elevado para degradar o corante.

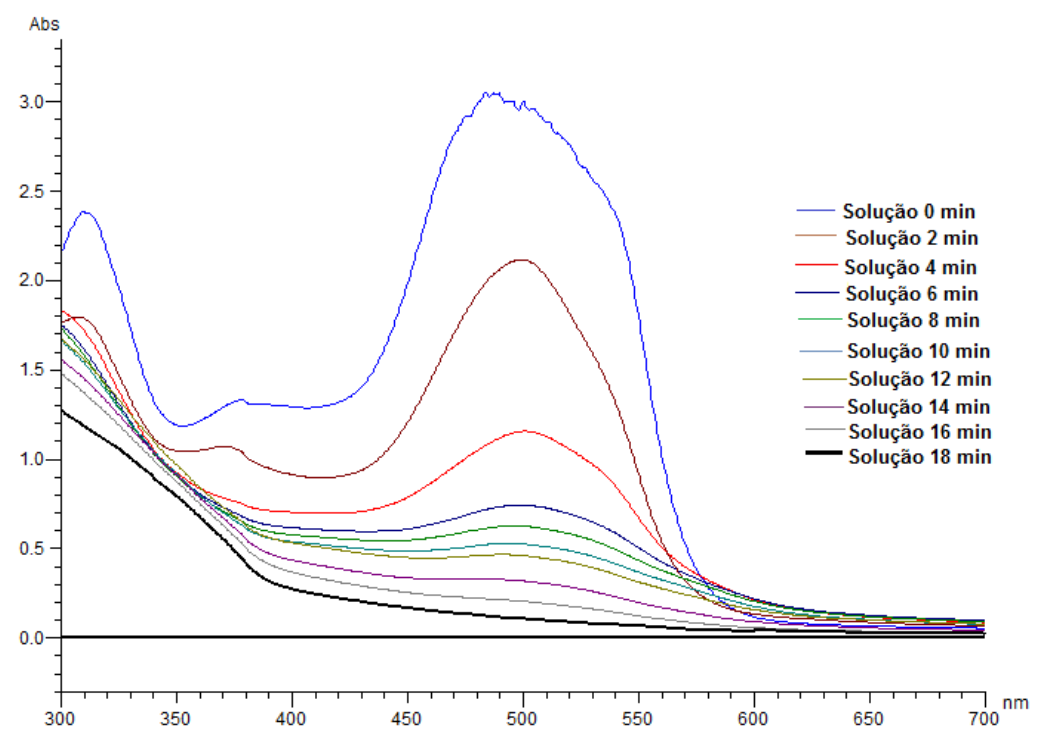

Figura 3 - Curva de calibração para uma faixa de concentrações entre 0,02 e $0,2 \mathrm{ppm}$.

\subsection{Efeito do Fluxo de Alimentação}

Conforme a Figura 4, são apresentados os resultados provenientes para o estudo da variação do fluxo do eletrólito através do reator. Aplicou-se uma variação de 300,600 e $800 \mathrm{~L} / \mathrm{h}$ para avaliação da degradação do corante, aplicou-se um potencial fixo de $3.5 \mathrm{~V}$ e uma concentração de cloreto de sódio de $0.6 \mathrm{~mol} / \mathrm{L}$. Neste contexto, pode-se verificar que a variação de fluxo não influencio na degradação do corante o que indicou um processo controlado por transferência de carga. 


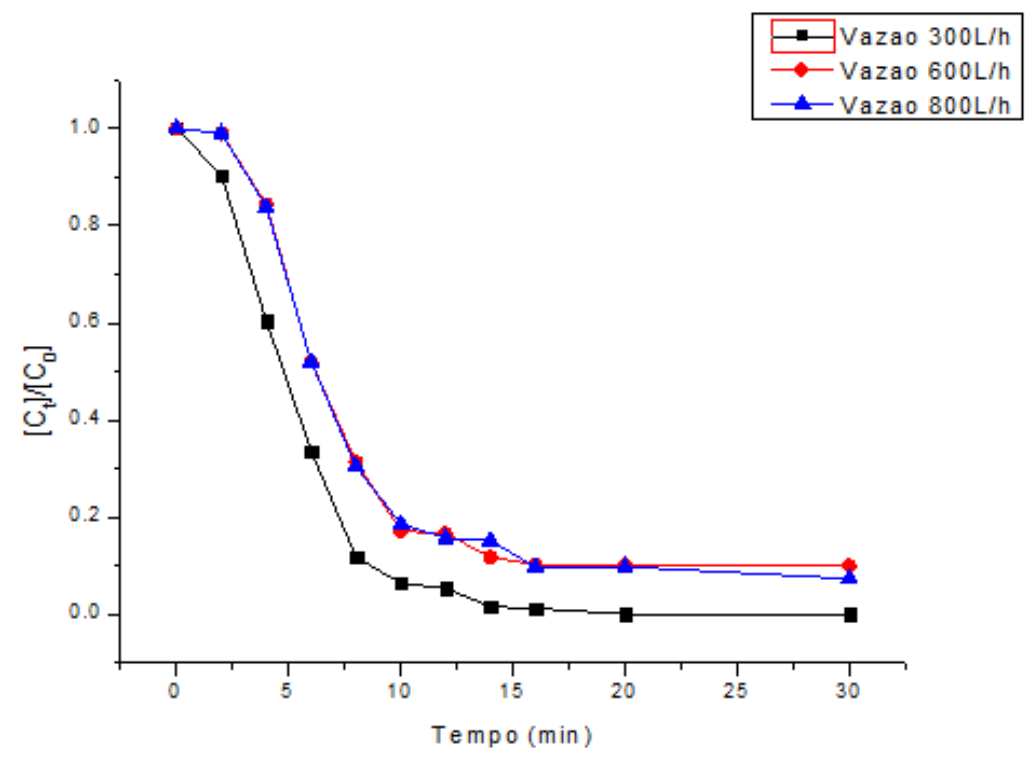

Figura 4 - Variação da concentração normalizada do corante em função do tempo para diferentes vazões, $T=25 \pm 1^{\circ} C,(\mathrm{ddp})=3,5 \mathrm{~V}$.

\subsection{Taxa de Degradação}

Um dos principais parâmetros de eficiência do processo é a taxa de remoção $X_{r}$ em relação ao tempo de eletrólise, e é calculada experimentalmente através da seguinte relação:

$$
X_{r}=\left(1-\frac{C\left(t_{0}\right)}{C_{(t)}}\right)
$$

Conforme a Figura 5, para concentrações de 1,2 e 2,0 $\mathrm{g} / \mathrm{L}$, a curva apresenta um valor máximo de 99,47\% de conversão para um tempo de eletrólise de 20 minutos. Com esses valores entende-se que após os primeiros 18 minutos de eletrólise indireta, a taxa de degradação alcançou seu valor máximo de conversão, estabilizando o processo. Também é perceptível que um aumento na concentração de cloreto de sódio de 1,2 para $2,0 \mathrm{~g} / \mathrm{L}$ não influenciou de maneira significativa a aumentar a degradação do vermelho ácido, obtendo-se uma mesma taxa de degradação para tempos iguais de eletrólise. 


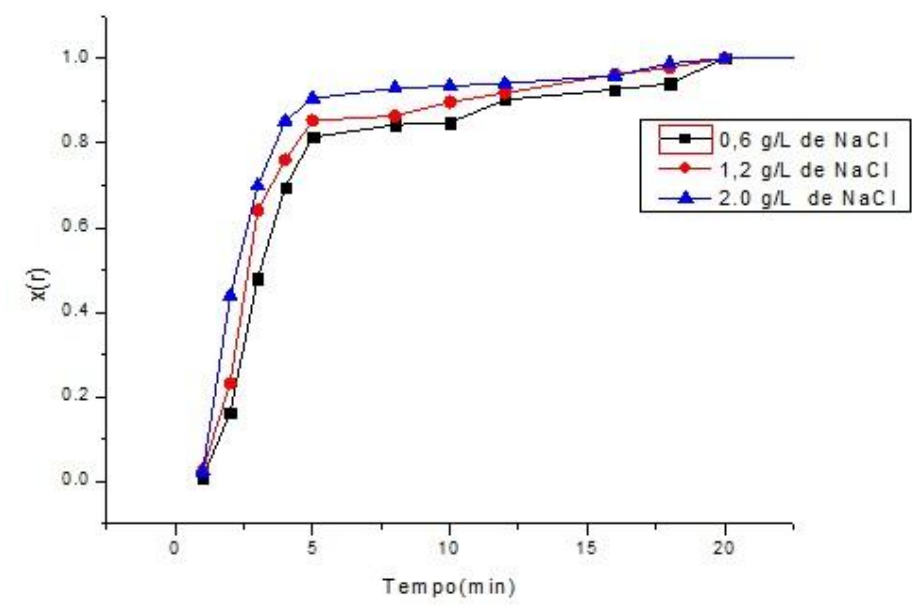

Figura 5 - Variação da taxa de conversão para diferentes concentrações de cloreto de sódio,

$$
T=25 \pm 1^{\circ} C,(\mathrm{ddp})=3,5 \mathrm{~V} \text {. }
$$

\section{Conforme A}

5 mostra os perfis das curvas das taxas de conversão experimentais comparadas com a curva obtida com o modelo teórico PFR utilizado. Verifica-se que a taxa de redução do corante não depende da vazão, utilizou uma vazão intermediária $\left(0,01 \mathrm{~m}^{3} / \mathrm{min}=600 \mathrm{~L} / \mathrm{h}\right) \mathrm{e}$ encontrou um coeficiente de transferência de massa é igual a $3.28 \times 10^{-6} \mathrm{~m} / \mathrm{min}$. Dessa maneira, com o valor do coeficiente de transferência conhecido, podemos usar equações de projeto encontradas na literatura para compararmos os percentuais de remoção obtidos experimentalmente com os percentuais calculados pelas expressões matemáticas advindas de um balanço de massa para um reator de fluxo pistão (PFR) com reciclo de eletrólito (WALSH, 1993). A equação 3 expressa o percentual de remoção em função do tempo para um reator PFR com reciclo.

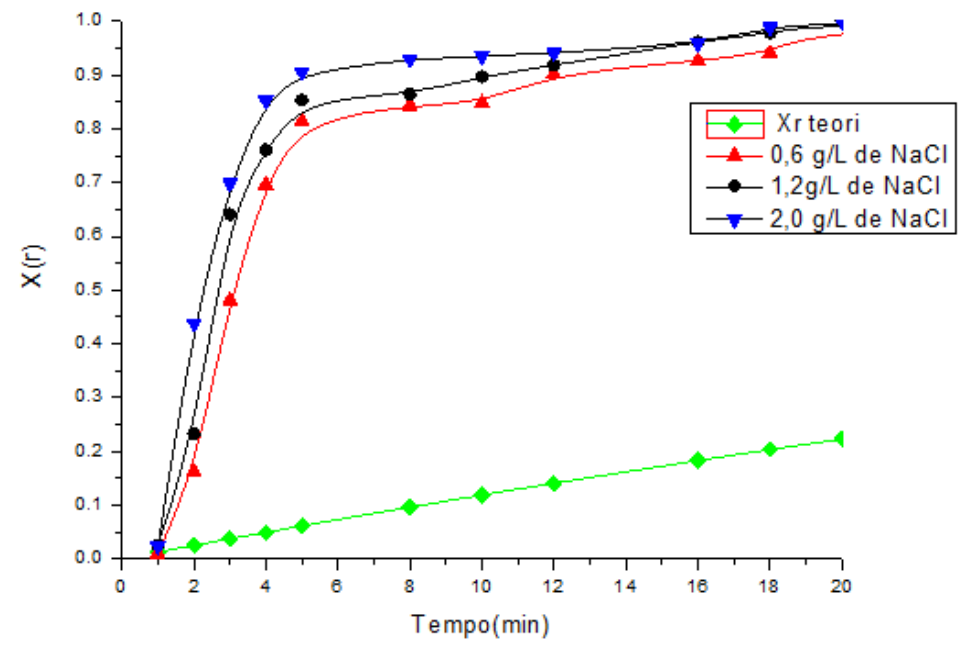

Figura 6 - Variação da taxa de remoção experimental e teórica, $T=25 \pm 1^{\circ} C$, $(\mathrm{ddp})=3,5 \mathrm{~V}$. 
Observa-se que o modelo da taxa de degradação do corante vermelho ácido não segue o modelo estabelecido teoricamente para um reator PFR sob-regime difusional, o que indica que a eletrooxidação do corante é regida por uma reação faradaica, ou seja, que depende da cinética de transferência de carga.

\subsection{Efeito da Variação do Potencial}

Com o objetivo de estudar a influência do potencial na eletrólise, as degradações do corante foram realizadas, utilizando três potencias $(3,5,4,0$ e 5,0V).

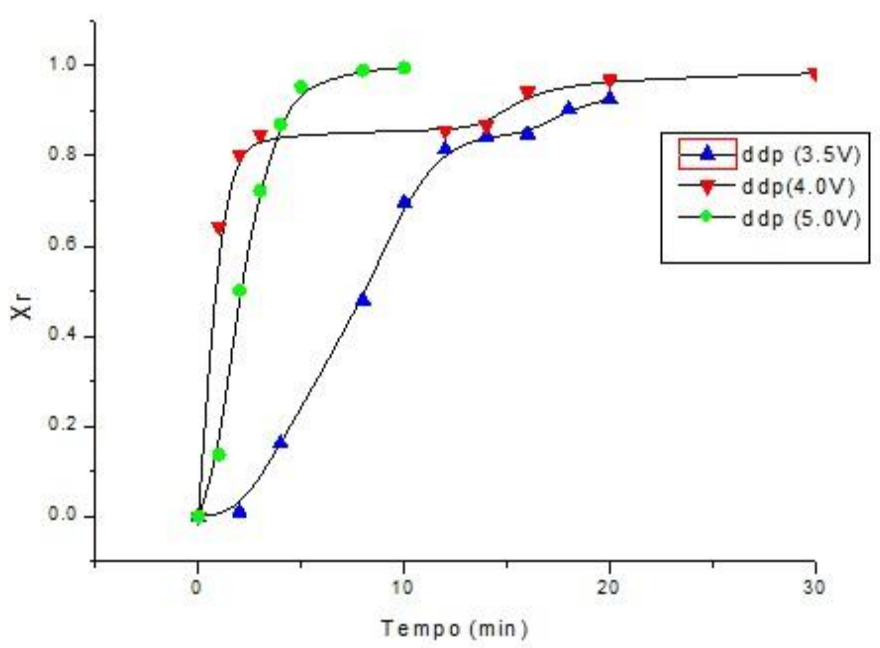

Figura 7 - Taxa de redução do corante para diferentes potenciais de (3,5, 4.0 e 5,0V), $T=25 \pm l^{\circ} \mathrm{C}, 0,6 \mathrm{~g} / \mathrm{L}$ de $\mathrm{NaCl}$.

Aplicando-se uma diferença de potencial (ddp) de $3.5 \mathrm{~V}$, ocorreu uma remoção de cor de 92,64\% em 20 minutos de eletrólise, para um potencial (ddp) de $4.0 \mathrm{~V}$, ocorreu uma remoção de cor $96,80 \%$ para o mesmo tempo de eletrolise, aumentando ainda mais o potencial (ddp) para 5,0V ocorreu uma remoção de cor de 99,47\%. Observa-se que o aumento do potencial na interface promove uma maior troca de cargas na interface do semicondutor-efluente, isso significa que o aumento do potencial na interface, gera uma maior intensidade de corrente; aumentando a redução percentual da concentração do corante, alcançando mais rapidamente a um valor máximo em torno de 99,47\%. Este fenômeno ocorre devido tanto ao aumento da cinética de oxidação direta como daquela provocada pelo aumento do gás cloro (agente oxidante) gerado.

\section{CONCLUSÃO}

Ao se comparar a taxa de remoção experimental com o modelo teórico, Conclui-se que a eletrooxidação do corante é regida pela cinética de transferência de carga e que mudanças na hidrodinâmica do sistema não influencia consideravelmente na remoção do corante vermelho ácido. Sendo assim, ocorreu uma redução 99,47 \% para um potencial de 5,0 V para um tempo de eletrólise de aproximadamente 10 minutos. 


\section{NOMENCLATURA}

$C_{\text {corante }}(t=0)=$ Concentração média antes da eletrólise no comprimento de onda de máxima absorção do corante vermelho ácido.

$C_{\text {corante }}(t)=$ Concentração média após a eletrólise no tempo t no comprimento de onda de máxima absorção do corante vermelho ácido.

$C_{t_{0}}=$ Concentração inicial do corante.

$C_{t}=$ Concentração em um tempo $t$.

$\mathrm{X}_{\mathrm{r}}=$ Taxa de remoção.

$I_{d}^{g}=$ Intensidade de corrente limite difusional na presença de bolhas de gás (A).

$n=$ número de elétrons envolvidos na reação.

$F=$ constante de Faraday $\left(\mathrm{C} / \mathrm{mol}^{-1}\right)$.

$\Delta C=$ variação de concentração do íon ferricianeto $\left(\mathrm{mol} / \mathrm{m}^{3}\right)$.

$V_{c}=$ Volume do compartimento Anódico $\left(\mathrm{m}^{3}\right)$.

$t=$ tempo de eletrólise $(s)$.

$\overline{K_{d}^{g}}=$ coeficiente médio de transferência de massa combinado com a influência fluidodinâmica em presença de gás $(\mathrm{m} / \mathrm{min})$.

$C=$ concentração média do ferricianeto entre o inicio e o final da eletrolise $\left(\mathrm{mol} \cdot \mathrm{m}^{-3}\right)$.

\section{REFERÊNCIAS}

ALBUQUERQUE I. L. T. Influência da Geometria Catódica na Transferência de Massa e Potencial Catódico durante a eletrólise da Água em meio alcalino, MsC UFCG UAEQ, Campina Grande - PB, 2006.

ELSENIER, C. Transfert de matiére et distribution du potential et du courant sur des électrodes de métal déployé, en presence de bulles electro- engendreés. Tese de Doutorado pela Universidade de Rennes 1, França 1984.

LIMA, J de F, Avaliação eletroquímica da espuma níquel G60 e placa de aço perfurada 316 objetivando sua utilização na eletrorrecuperação do ouro lixiviado proveniente de Princesa Isabel-PB. Dissertação de Mestrado pela Universidade Federal de Campina Grande - UFCG, 2013.

JOVIC, M.; STANKOVIC, M. D.; MANOJLOVIC, D.; ANDELKOVIC; MILIC, A. Study of the Electrochemical Oxidation of Reactive Textile Dyes Using Platinum Electrode. Faculty of Chemistry, University of Belgrade, Student Square 12-16, 11158 Belgrade, Serbia, p. 168-183, 2013.

WALSH, F. C. A Firsth course in electrochemical engineering. The eletrochemical consultancy, ECC, $1^{\text {a }}$ Ed., Romsey. England. 1993. 